\title{
Gondeschapur - eine Medizinschule aus dem 6. Jahrhundert n. Chr.
}

Von Friedrun R. Hau

Die theoretische und praktische Ausbildung des angehenden Arztes bestimmt in entscheidender Weise Erfolg oder Mißerfolg bei seiner späteren Tätigkeit - und dies gilt nicht erst heute.

Wegweisend für die berühmten medizinischen Ausbildungszentren des islamischen Mittelalters in Bagdad, Kairo und Damaskus war die Medizinschule von Gondeschapur, die um die Mitte des 6. Jahrhunderts in der südpersischen Provinz Chûzistân gegründet worden ist ${ }^{1}$.

Wenn uns auch über die vorislamische (vor $736 \mathrm{n}$. Chr.) und frühislamische Periode bisher kaum Quellen bekannt sind und daher Spekulationen reichlich Nahrung gefunden haben, so können wir aus Einsprengseln innerhalb einiger Ärztebiographien aus der frühen Abbasidendynastie (750-1258), insbesondere von nestorianischen Christen in und aus Gondeschapur, sowie aus Geschichten über ihre den Muslimen recht seltsam anmutenden Heilmethoden wenigstens in Umrissen ein Bild nachzeichnen.

Die sassanidische Medizinschule in Gondeschapur hat nämlich einen wesentlichen Beitrag zur Wahrung der antiken Medizin und zur Förderung der Medizin für das spätere islamische Mittelalter geleistet. Ihr Vorbild war die traditionsreiche Medizinschule in Alexandria. Im Hinblick auf die Ausbildung des medizinischen Nachwuchses können Alexandria und Gondeschapur als die einzigen Hochburgen späthellenistischer Prägung von Rang bezeichnet werden.

In Alexandria wirkten bereits im dritten vorchristlichen Jahrhundert im sogenannten «Museion», dem von den Ptolemäern gegründeten Forschungszentrum, bedeutende Ärzte wie Herophilos und Erasistratos ${ }^{2}$; an der menschlichen Leiche führten sie anatomische und pathologische Sektionen durch und experimentierten am Tier; sie unterschieden bereits motorische und sensible Nerven sowie Arterien und Venen bei den Blutgefäßen. Herophilos hat die Pulsfrequenz mit der Wasseruhr gemessen, und Erasistratos wollte dem Geheimnis des Stoffwechsels auf die Spur kommen, indem er bei Geflügel die Nahrungsmenge vor und nach der Einnahme wog und den Gewichtsverlust beim Exkrement als «unmerkliche Transpiration» bezeichnete.

Während Alexandria auf eine alte Tradition zurückblicken kann, fließen die Nachrichten über die Gründung Gondeschapurs sehr spärlich.

Barhebraeus berichtet in seiner «Kurzgefaßten Dynastiengeschichte ${ }^{3}{ }^{3}$ daß 
der Kaiser Aurelianus während seiner Regierungszeit (270-275) mit Schapur I. (reg. 242-272) einen Waffenstillstand geschlossen habe. Zur Besiegelung soll Aurelianus Schapur seine Tochter zur Frau gegeben haben; auf ihrer Reise nach Persien befand sich im Gefolge auch eine Gruppe griechischer Ärzte, die für die Propagierung der hippokratischen Medizin sorgen sollten. Schapur soll dann für seine neue Frau eine Stadt - nach dem Vorbild von Byzanz - erbaut und sie « Ğundaisâbûr» genannt haben ${ }^{4}$.

Schenkt man Barhebraeus Glauben, müßte Gondeschapur innerhalb der Jahre 270-272 errichtet worden sein, da Schapur I. nur bis zum Jahre 272 regiert hat, der römische Kaiser Aurelianus aber erst im Jahre 270 an die Macht gelangt ist.

Schapur soll gemäß anderen Quellen in Gondeschapur eine Akademie gegründet haben $^{5}$; dies bezweifelt Karl Hummel nachdrücklich, da seiner Meinung nach erst zur Regierungszeit des Königs Chosrau I. Anuschirwân (reg. 531-579) «auch nur annähernd derartige Vorbedingungen für die Entstehung der Akademie vorhanden » gewesen seien ${ }^{6}$.

Ob nun eine Akademie schon im dritten oder erst im sechsten Jahrhundert in Gondeschapur gegründet worden ist, von allen unbestritten ist die Tatsache, daß der zuvor erwähnte Chosrau I. Anuschirwân, der als Förderer der Wissenschaften beschrieben wird, den Bau einer Medizinschule veranlaßt hat; diese soll mit dem bereits vorhandenen Krankenhaus kooperiert haben ${ }^{7}$. Die Ärzte waren überwiegend nestorianische Christen ${ }^{8}$, die hier im persischen Raum eine Zuflucht vor den Verfolgungen der Byzantiner gefunden hatten.

Im Hinblick auf den medizinischen Lehrplan dieser Medizinschule hat Max Meyerhof in einer ausführlichen Studie den Wanderweg philosophischer und medizinischer Lehrstoffe von Alexandria über Nisibis, Edessa und Gondeschapur bis nach Bagdad nachgezeichnet ${ }^{9}$.

So wurden vermutlich in Gondeschapur für den theoretischen Unterricht der Medizinstudenten die sogenannten «Summaria Alexandrinorum» verwendet, eine von Alexandriner Ärzten angefertigte Synopse von sechzehn Galen-Schriften mit dem griechischen Titel «tois eisagoménois», über die aber in der griechischen Überlieferung seltsamerweise nichts berichtet wird ${ }^{10}$.

Aus diesem Umstand resultieren nach Owsei Temkins Meinung «zwei Möglichkeiten der Erklärung. Entweder die feste Kanonisierung erfolgte in Alexandrien erst nach Sergius, also gegen Ende des 6. bzw. im 7. Jahrhundert, und die Kunde davon gelangte vielleicht über den von Meyerhof aufgedeckten Weg direkt nach Bagdad. Oder die feste Kanonisierung erfolgte erst durch die Syrer und Araber, während man in Alexandrien sich niemals starr an einen sechzehnteiligen Kanon hielt. ${ }^{11}$ Auf jeden Fall erfolgte die Kanonisierung nach Temkin nicht vor der 
Mitte des 6.Jahrhunderts. Denn der Priester und Archiater Sergios aus Resch cAinâ (gest. 536; diese Stadt heißt auch Theodosiopolis) ${ }^{12}$, der in Alexandria studiert hatte, hat insgesamt wohl vierundzwanzig galenische bzw. pseudogalenische Schriften ins Syrisch-Aramäische übersetzt, indessen nur dreizehn von den sechzehn Schriften der sogenannten «Summaria Alexandrinorum»; es fehlen das erste Buch über «die Schulen», das kleine Buch über «den Puls» und «die Erhaltung der Gesundheit».

Am liebsten möchte Temkin die Schaffung dieser sechzehnteiligen GalenSynopse «als ein Werk der Syrer und Araber ${ }^{13}$ betrachten. Dann wäre auch geklärt, weshalb in der griechischen Überlieferung überhaupt keine Spur dieses Alexandriner Kanons auffindbar ist.

Die zuvor erwähnte syrische Übersetzung der Galen-Schriften durch Sergios ist sicherlich gleich in der zweiten Hälfte des 6. Jahrhunderts in der jungen Medizinschule Gondeschapurs für den theoretischen medizinischen Unterricht verwendet worden. Denn die hier praktizierenden nestorianischen Ärzte werden sich wohl ihrer Muttersprache - des Syrisch-Aramäischen - bei der Unterweisung bedient haben ${ }^{14}$.

Im Jahre 736 wurde die Stadt Gondeschapur durch die arabische Invasion (glücklicherweise unzerstört) eingenommen. Als um das Jahr 862 eine neue Stadt buchstäblich aus dem Wüstensand gestampft und dieses Bagdad zur neuen Kalifenresidenz erkoren wurde, da fiel plötzlich auf die versierten syrischchristlichen Ärzte im nahe gelegenen Gondeschapur (nur ein paar Tagereisen entfernt im Vergleich zum fernen Damaskus vorher) gleichsam das Rampenlicht der Weltbühne, und sie genossen bei den neuen Machthabern der AbbasidenDynastie hohes Ansehen. Nun sprudeln auch die Nachrichten über Gondeschapur, seine Ärzte und ihre andere - weil christliche - Lebensweise aus den arabischen Quellen der Ärztebiographen.

Als erster Kalif der Abbasiden-Dynastie (750-1258) ließ Manșûr (reg. 754-775) im Jahre 765 Dschurdschîs ibn Bachtîschuc ${ }^{c}$ (gest. 768) ${ }^{15}$, den damaligen Direktor des Krankenhauses von Gondeschapur, zur Behandlung eines Magenleidens nach Bagdad holen. Der Arzt Ibn abî Ușaibica (gest.1270) berichtet in seinen «Quellen der Ärztebiographien», daß der Kalif äußerst erstaunt war, als ihn der Arzt aus Gondeschapur in arabischer und persicher Sprache begrüßte ${ }^{16}$, weil bekanntlich die Leute in Gondeschapur ihre eigene Sprache hatten, das SyrischAramäische.

Dschurdschîs hat ein bekanntes medizinisches Kompendium in aramäischer Sprache - daher «Kunnâsch» genannt - verfaßt, das später Johannitius ins Arabische übersetzt hat ${ }^{17}$; Rhazes zitiert es mehrmals in seiner voluminösen 
medizinischen Exzerptensammlung «Kitâb al-Ḥ̂wî̀, die dem Abendland in lateinischer Übersetzung als «Liber Continens» bekannt geworden ist.

Ein Enkel des zuvor erwähnten Dschurdschîs, nämlich Dschibra'îl ibn Bachtîschûc ${ }^{\mathrm{c}}$ (gest. 827/28) ${ }^{18}$, wurde im Jahre 805 Leibarzt des aus Tausendundeiner Nacht hinlänglich bekannten Kalifen Hârûn ar-Raschîd und diente darnach in gleicher Funktion bei den Kalifen Amîn und Ma'mûn. Dschibra'îl ist durch viele Geschichten berühmt geworden; eine davon soll im folgenden wörtlich übersetzt werden, weil sie ein Streiflicht nicht nur auf das höfische Leben der damaligen Zeit wirft, sondern auch auf die ärztliche Handlungsweise eines angesehenen nestorianischen Arztes und somit die theoretische und praktizierte galenische Humoralphysiologie und -pathologie trefflich in gedrängter Form anklingen läßt. «Eines Tages räkelte sich die Geliebte (Hârûn ar-) Raschîds und erhob ihre Hand. So blieb sie ausgestreckt, ohne daß sie sie zurücknehmen konnte. Die Ärzte behandelten sie, indem sie (die Hand) einsalbten und einölten. Aber es nutzte nichts.

Da sagte Raschîd zu Dschacfar ibn Yahyâ: 'Dieses Mädchen wird wohl seine Krankheit behalten.' Dschacfar erwiderte ihm : 'Ich habe einen erfahrenen Arzt es ist Ibn Bachtîschû́c. Bitte ihn, zu dir zu kommen, und sprich ihn auf die Bedeutung dieser Krankheit an! Vielleicht weiß er einen Kniff für ihre Behandlung.'

Da ließ Raschîd den Arzt holen. Als er erschienen war, fragte ihn Raschîd: 'Wie heißt du?' Er antwortete: 'Dschibra'1̂l.' Der Kalif fragte ihn: 'Was verstehst du von der Heilkunde?' Dschibra'îl erwiderte: 'Ich kann Heißes kühlen und Kühles erwärmen, Trockenes kann ich feucht machen und trocknen das Feuchte, was von der Natur (des Patienten) abweicht.' Da brach der Kalif in Lachen aus und meinte: 'Das ist (wohl) das Äußerste dessen, was man in der Medizin an Fertigkeit benötigt.'

Danach berichtete ihm der Kalif von dem Gesundheitszustand des Mädchens. Dschibra'îl sagte zu ihm: 'Wenn du mir nicht zürnst, o Fürst der Gläubigen, so habe ich eine List dafür.' Raschîd fragte: 'Was ist das ?' Dschibra'îl antwortete: 'Du sollst das Mädchen hierher in die Gegenwart der Leute herausholen [was absolut gegen die Sitte war!], damit ich das Beabsichtigte mit ihr durchführen kann. Dabei darfst du weder langsam noch schnell zornig werden.'

Da befahl Raschîd, das Mädchen zu holen. So kam sie heraus. Als Dschibra'îl sie erblickt hatte, eilte er gesenkten Hauptes zu ihr und ergriff ihren Rocksaum, als ob er sie entblößen wollte. Da wurde das Mädchen verwirrt. Vor lauter Scham und Verwirrung wurden ihre Gliedmaßen gelöst. Sie streckte ihre Hand nach unten aus und hielt den Rocksaum fest. Da sprach Dschibra'îl: 'Sie ist ge 
nesen, o Fürst der Gläubigen.' Raschîd forderte das Mädchen auf: 'Strecke deine rechte und deine linke Hand aus!' Sie tat es. Da wunderten sich Raschîd und alle Anwesenden. Dann ließ der Kalif Dschibra'îl sofort 500000 Dirham bringen. Er mochte ihn gern und ernannte ihn zum 'Chef aller Ärzte'.

Als Dschibra'îl nach der Ursache der Krankheit gefragt wurde, antwortete er: 'In die Glieder dieses Mädchens ist während des Beischlafs ein feiner Bestandteil durch die Bewegung und die Ausbreitung von Wärme geflossen. Weil unversehens Ruhe auf die Bewegung beim Beischlaf eintrat, gefror der Rest im Innern der Nerven. Nichts vermag den Rest aufzulösen, es sei denn eine ähnliche Bewegung. So wandte ich eine List an, damit sich die Wärme bei ihr ausbreiten und der Rest auflösen würde. So genas sie. Dies ist ein Kunstkniff zur Genesung.'

Deshalb heißt es im Buch über «die Prüfung des Arztes»: 'Es ist notwendig, daß ein Arzt wachsam und scharfsinnig ist und die Fähigkeit zur Anwendung des Analogieschlusses besitzt, damit er die Methoden zur Behandlung nach eigenem Ermessen ableiten kann.' $\gg 19$

In der geschilderten Weise wirkte die berühmte Ärztefamilie Bachtîschû $\hat{u}^{c}$ fast drei Jahrhunderte lang in Gondeschapur in leitender Stellung am Krankenhaus oder in Bagdad am Kalifenhof.

Dschibra'îl soll für den Abbasidenkalifen Ma'mûn eine «Abhandlung über Essen und Trinken», ferner eine «Einführungsschrift in die Logik» und eine Schrift «über den Coitus» abgefaßt haben ${ }^{20}$.

Aus einer anderen Ärztefamilie in Gondeschapur stammt Yûhannâ ibn Mâsawaih (777-857), der persich «Mâsûye» ausgesprochen wird und dessen latinisierter Name deshalb «Mesuë» lautet ${ }^{21}$. Yûhannâ «stand im Dienst der Kalifen Hârûn ar-Raschîd, Amîn, Ma'mûn und blieb dies bis in die (Regierungs-) Tage des Mutawakkil (reg. 847-861) ».22

Yûhannâ war ein bekannter Privatgelehrter; in seinen Disputationsrunden für Ärzte, muslimische Theologen und Philosophen ging es äußerst kultiviert zu, weil er dafür Vertreter sämtlicher antiker Disziplinen in sein Haus einzuladen pflegte $^{23}$. Yûhannâ war schon als Kind mit seinem Vater von Gondeschapur nach Bagdad umgesiedelt, nachdem sein Vater über dreißig Jahre lang am Krankenhaus in Gondeschapur tätig gewesen war; aber Dschibra'îl ibn Bachtîschû́ $\hat{u}^{\mathbf{c}}$, der in seiner Funktion als vom Kalifen ernannter «Oberarzt» offenbar auch für die Krankenhäuser zuständig war, hatte ihn wegen einer doppeldeutigen Äußerung kurzerhand entlassen. Daraufhin war Yûhannâs Vater nach Bagdad gereist, um sich persönlich bei Dschibra'îl zu entschuldigen; doch dieser ließ ihn lange Zeit nicht vor. In einem Wesir des Kalifen Hârûn ar-Raschîd, dessen am Auge erkrankten Diener Yûhannâs Vater erfolgreich behandelt hatte, fand er 
einen Gönner und dank dessen Fürsprache wie auch dank seiner hervorragenden Fähigkeiten als Augenarzt schließlich sogar eine Stellung am Hofe und damit endlich wieder einen gesicherten Lebensunterhalt ${ }^{24}$.

Einige arabische Quellen berichten, daß «Hârûn ar-Raschîd den Nestorianer Yûhananâ ibn Mâsawaih mit der Übersetzung antiker medizinischer Schriften beauftragt hat, nachdem der Kalif diese in Ankara, Ammuriya und den übrigen Städten der Byzantiner während der Eroberung und Vereinnahmung dieser Städte durch die Muslime erlangt hatte. Hârûn setzte ihn als Kustos für die Übersetzungstätigkeit ein. ${ }^{25}$ Yûhananna fertigte auch selbst Übersetzungen aus der griechischen in die arabische Sprache an, von denen uns aber keine erhalten geblieben ist.

Unter seinen zahlreichen Schriften ist für den Medizinhistoriker vielleicht der «Kitâb al-muschadschdschar» am interessantesten; denn in diesem frühen Lehrbuch der Medizin wurde der Stoff in Form eines Stammbaumes ausgebreitet; bisher gibt es indessen noch keine Edition dieses Werkes ${ }^{26}$.

Yûhannâ scharte in seinem langen, acht Jahrzehnte umfassenden Leben zahlreiche Schüler um $\operatorname{sich}^{27}$. Sein bedeutendster Schüler ist zweifelsfrei Hunain ibn Ishâq (808-873, nach anderen 877; latinisiert «Johannitius» ${ }^{28}$. Auch Ḥunain ist nestorianischer Christ, aber gebürtig aus der Stadt Ḥîra (Herat) in Ostpersien und Sohn eines Apothekers. Schon in jungen Jahren liebte er die Wissenschaft und ging deshalb nach Bagdad; dort studierte er bei Yûhannâ ibn Mâsawaih und fiel diesem wegen seiner vielen, oft bohrenden Fragen auf. Einmal wurde Yûhannâ bei einer Frage so wütend, daß er sich zu der rhetorischen Frage hinreißen ließ: «Was haben eigentlich die Leute von Ḥîra mit der Medizin zu tun ? ${ }^{29}$ Die näheren Umstände und den Hintergrund zu diesem Ausspruch schildert ausführlich Ibn abî Ușaibica in seinen «Ärztebiographien»:

«Hunain pflegte unvermittelt Fragen zu stellen, deren Beantwortung Yûḥannâ manchmal schwerfiel. Außerdem brachte der Umstand, daß Ḥunain den Nachkommen der Geldwechsler aus (der Stadt) Ḥîra entstammte, ihn dem Herzen Yûḥannâs nicht näher, während doch die Leute von Gondeschapur Gebildete waren, deren Ärzte sich von den Leuten aus Hîra (verächtlich) abwandten. Sie (d.h. die Leute von Gondeschapur) haßten es, wenn Söhne von Kaufleuten in ihre Kunst eindringen wollten. ( $^{30}$

Nach dieser Demütigung durch seinen Lehrer vor der Klasse verließ Hunain Bagdad und begab sich auf Reisen für mindestens zwei Jahre durch das byzantinische Reich, um selbst seine Griechisch-Kenntnisse weiter vertiefen zu können $^{31}$. Nach seiner Rückkehr stellte er sich in Bagdad bei dem zuvor erwähnten Dschibra'̂̂l ibn Bachtîschû́ ${ }^{\mathrm{c}}$ vor; ein Arzt mit Namen Yûsuf erzählt: 
«Damals trat ich eines Tages bei Dschibra'îl ibn Bachtîschûc ein und fand bei ihm Ḥunain vor, der ihm gerade einige griechische Passagen aus einem der Bücher Galens 'über die Anatomie' übersetzte, wobei ihn Dschibra'̂̂l ehrerbietig ansprach. Ich empfand das, was ich beobachtet hatte, als bedeutend. Da sagte Dschibra'îl zu mir: 'Du darfst meine Haltung gegenüber diesem jungen Mann nicht übertrieben finden. Denn bei Gott, wenn er (d.h. Gott) ihm ein langes Leben gewährt, wird er wahrhaftig Sergios überstrahlen - das ist Sergios von

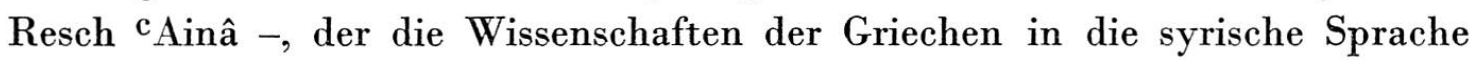
übersetzt hat.' ${ }^{32}$

Ibn al-Qiftî berichtet in seiner «Geschichte der Gelehrten und Ärzte», daß nach Hunain auch der Arzt Yûsuf wieder hinausgegangen sei. Da habe Hunain ihn schon erwartet und ihn einiges über seinen ehemaligen Lehrer Yûhanna gefragt. Dann habe er ihn gebeten, eine Kopie einiger Abschnitte aus den «Summaria Alexandrinorum » Yûhannâ auszuhändigen. Yûhannâ bat seinerseits den Arzt Yûsuf um Vermittlung. Schließlich versöhnten sich der Lehrer und der ehemalige Schüler ${ }^{33}$.

Von dieser Zeit an widmete sich Ḥunain der Übersetzungstätigkeit und hat selber über hundert Schriften von Galen, Hippokrates und anderen griechischen Ärzten nach griechischen Handschriften ins Syrisch-Aramäische bzw. Arabische übersetzt. Dies hing davon ab, ob sein Auftraggeber ein Christ oder ein Muslim war.

Seine sehr modern anmutende Übersetzungsmethode legt er in seinem sogenannten «Sendschreiben» (das Gotthelf Bergsträsser kritisch ediert und ins Deutsche übersetzt hat) nach der Inhaltsangabe des vierzehn Teile umfassenden Buches «Über das Heilverfahren» wie folgt dar:

«Salmawaih hatte mich angetrieben(?), für ihn diese zweite Hälfte zu verbessern, und hatte sich (dabei) eingebildet, dies werde leichter und besser sein, als sie (neu) zu übersetzen. So kollationierte er mit mir ein Stück des siebenten Teils, indem er das Syrische in der Hand hatte und ich das Griechische und er mir das Syrische vorlas und ich jedesmal, wenn mir etwas vom Griechischen Abweichendes aufstieß, ihm dies mitteilte und er dann zu verbessern begann. Schließlich aber wurde ihm die Sache zu viel, und es wurde ihm klar, daß es bequemer und erfolgreicher sein würde, von vorn (neu) zu übersetzen, und daß dabei das Ergebnis geordneter sein würde.» ${ }^{34}$

Hunain hat dann die acht Teile der zweiten Hälfte (Teile 7-14) vollständig übersetzt; sie sind aber bei einem Brand mit allen Kopien vernichtet worden. Hunain fährt dann in seinem «Sendschreiben» fort:

«Nach Jahren habe ich dann das Buch von Anfang an für Bohttîšô ibn Gibrîl 
übersetzt, wobei ich von seinen acht letzten Teilen eine Anzahl Handschriften auf griechisch hatte; so habe ich diese kollationiert, aus ihnen ein korrektes Exemplar hergestellt und es mit der größten mir möglichen Genauigkeit und Eleganz übersetzt. Was dagegen die sechs ersten Teile anlangt, so war ich nur auf eine einzige Handschrift von ihnen gestoßen, und dies war obendrein eine sehr fehlerhafte Handschrift. Deshalb war es mir nicht möglich, diese Teile ganz so herzustellen, wie erforderlich. Dann stieß ich auf eine weitere Handschrift, kollationierte sie und verbesserte, was mir (mit ihrer Hilfe) zu verbessern möglich war; ich würde(?) es (das Buch) aber gern (?) (noch) ein drittes Mal kollationieren, wenn mir eine dritte Handschrift zufällt. Die Handschriften dieses Buches auf griechisch sind nämlich wenig zahlreich, und zwar, weil es nicht zu denen gehörte, die in der alexandrinischen Schule gelesen wurden. ${ }^{35}$

Wie in diesem Falle Ḥubaisch, so besorgten oft Hunains Schüler die Übersetzung ins Arabische nach der syrischen Vorlage ihres Meisters, der dann lediglich die Mängel ihrer Übersetzung revidierte ${ }^{36}$.

Da Ḥunain in seinem «Sendschreiben» jeweils nach der kurzen Inhaltsangabe eine bereits vorhandene syrische Übersetzung vermerkt, kannte er die syrischen Versionen der 24 Galenschriften durch Sergios von Resch ${ }^{c_{A i n a ̂}}$; doch genügten sie in den meisten Fällen nicht seinen hohen Anforderungen, wofür die vorige Geschichte mit Salmawaih ein beredtes Beispiel liefert ${ }^{37}$.

Hunain und seiner Übersetzerschule kommt das hohe Verdienst zu, alle wichtigen medizinischen und philosophischen Schriften antiker Gelehıter ihren meist nur einsprachigen muslimischen Nachbarn zugänglich und verständlich gemacht zu haben. Zur Befriedigung einer solchen wissenschaftlichen Übersetzung mußte Ḥunain die arabische Sprache so gestalten, daß «komplizierte abstrakte Sachverhalte in logisch klaren Bezügen» ausgedrückt werden konnten; dies gelang ihm durch die «Einführung analytischer Konstruktionen in die Syntax ». ${ }^{38}$ Manfred Ullmann bewertet «diese Sprachschöpfung» als «eine philologische Leistung ersten Ranges; und sie ist besonders hoch zu bewerten, da sie keine Unterstützung von Seiten der professionellen arabischen Philologen fand $»,{ }^{39}$ die nur die Beduinenpoesie, den Koran und den Hadît (d.i. die Traditionswissenschaft) erforschten.

Ḥunain hat außer den enorm vielen Übersetzungen und Bearbeitungen von Galen-Schriften und anderen antiken Werken auch eigene Abhandlungen verfaßt, wie den «Aderlaß», die «Erhaltung der Zähne und des Zahnfleischs», die «Kenntnis der Magenschmerzen und ihre Heilung», die «Behandlung der Wassersüchtigen», die «Entstehung des (Blasen-) Steines» und das «Bad», die uns aber nicht einmal in Handschriften erhalten sind, sondern fast durchweg nur in 
bruchstückhaften Zitaten des Rhazes in seinem «Kitâb al-Ḥâ̂î̀ («Liber Continens $")^{40}$.

Allein die «Einleitung in die Medizin» ist in ihrer frühen lateinischen Übersetzung unter dem Titel «Isagoge in artem parvam Galeni» zur Einführungsschrift für den Medizinstudenten schlechthin im Abendland geworden ${ }^{41}$; später ist sie in dem ersten Articella-Druck in Venedig im Jahre 1483 gleich am Anfang zu finden ${ }^{42}$.

Wer auch immer der Übersetzer des «Kitâb al-Mudchal fî ț-țibb » ins Lateinische gewesen sein mag, ob Marcus von Toledo oder ein anderer Übersetzer, unbestritten ist die prominente Rolle der «Isagoge Johannitii » als theoretischer Meilenstein am Anfang des Ausbildungsweges eines jeden angehenden Arztes.

Hunain hat auch für die Augenärzte ein Lehrbuch unter dem Titel «Zehn Abhandlungen über das Auge» abgefaßt ${ }^{43}$, das nach der Angabe des Arztes und Gewerbeaufsehers Schaizarî als Leitfaden für gewerbliche Überprüfung der Augenärzte dienen sollte ${ }^{44}$.

Anhand des Lebenslaufs einiger Ärzte der berühmten Familien Bachtîsch $\hat{u}^{\mathrm{c}}$ und Mâsawaih ist versucht worden, die in Gondeschapur praktizierte Medizin anzudeuten und die teils im privaten, teils im politischen Bereich liegenden Gründe für eine Übersiedlung in die neue Kalifenresidenz und somit die Weitergabe der im christlichen Gondeschapur gängigen Behandlungsmethoden an die meist staunenden muslimischen Ärzte am Kalifenhofe aufzuzeigen. Die angeführten Episoden machen offenbar, daß die nestorianisch-christlichen Ärzte aus Gondeschapur ein beträchtlich hohes Ansehen genossen haben; dies sollte sich erst zwei Jahrhunderte später zugunsten der muslimischen Ärzte ändern ${ }^{45}$.

Bis dahin erging es muslimischen Ärzten seitens ihrer Glaubensgenossen im Hinblick auf ihre Praxis schlecht. Ein beredtes Beispiel gibt der muctazilitische Theologe Dschâhiz ${ }^{46}$ in seinem satirischen «Buch über die Geizhälse», indem er als Fürsprecher eines Bagdader muslimischen Arztes die Misere anprangert: Für die Patienten sei er ein Muslim und deshalb mieden sie ihn; offenbar brächten sie einem nestorianischen Arzt mehr Vertrauen entgegen; dann müßte er einen aramäischen Vor- und Zunamen haben; ferner dürfte sein Obergewand nicht aus weißer Baumwolle, sondern aus schwarzer Seide sein, und letztlich dürfte er nicht arabisch sprechen, sondern die Sprache der Leute von Gondeschapur, damit seine Praxis nicht leer stände ${ }^{47}$.

Wann die Medizinschule in Gondeschapur zu funktionieren aufgehört hat, läßt sich nicht ausfindig machen. Wahrscheinlich wird schon das um das Jahr 800 unter dem Abbasidenkalifen Hârûn ar-Raschîd (reg. 786-809) errichtete Krankenhaus einige junge Ärzte aus Gondeschapur in Anbetracht des vielfältigen 
kulturellen Lebens in die Metropole gelockt haben ${ }^{48}$. Darnach wird der Kalif Ma'mûn (reg. 813-833) an seine um das Jahr 830 gegründete Übersetzer-Akademie, das sogenannte «Bait al-hikma», einige Gelehrte aus Gondeschapur geholt haben ${ }^{49}$.

Vermutlich wird die Medizinschule in Gondeschapur mangels Nachwuchses allmählich - wie ein Baum, der nicht mehr Wasser bekommt - zugrunde gegangen sein. Als letzte offizielle Nachricht aus der Medizinschule wird von Cyril Elgood die Veröffentlichung einer Pharmakopöe des Nestorianers Sâbûr ibn Sahl (gest. 869) im Jahre 869 angegeben ${ }^{50}$. Diese Pharmakopöe wurde im Osten des muslimischen Reiches für alle Apotheken und Krankenhäuser für verbindlich erklärt und stellt vielleicht die erste offizinell verordnete Pharmakopöe überhaupt dar.

\section{Anmerkungen}

${ }^{1} \mathrm{Zu}$ Gondeschapur s. Art. "Gondêshâpûr» in Encyclopaedia of Islam, New Edition II (1965) Sp. 1119b-1120b; Gottschalk (1971) S. 51f.; Sabra (1976) S. 182 b; Kunitzsch (1975) S. 274; Hossein Nasr (1976) Sp. 155 a; Watt (1974) S. 134f.

${ }^{2} \mathrm{Zu}$ Herophilos s. Dictionary of Scientific Biography VI (1972) Sp. 316b-319a und zu Erasistratos IV (1971) Sp. 382 b-386b. Bei Temkin (1932 S. 49) heißt es: «Ein dünner Pfad führte zwar von Alexandria direkt zu den Arabern über, der Hauptstrom floß jedoch über Syrien, vornehmlich über die Schule von Gondešapur»; Ackerknecht (1975) S. 63.

Herrn Prof. Dr. Nikolaus Mani danke ich für den freundlichen Hinweis auf Gustav Senn (1933), der in seiner «Entwicklung der biologischen Forschungsmethode in der Antike» die Verdienste des Herophilos (S. 135-141) und des Erasistratos (S. 141-150) ausführlich würdigt.

${ }^{3}$ Barhebraeus ist die latinisierte Namensform des Ibn al-c Ibrî (gest. 1286), der unter anderem auch Medizin studiert hat; er verfaßte eine «Dynastiengeschichte», von der uns heute aber nur der Auszug des Zauzanî (arab. «Muchtașar ta'rîch ad-duwal») zugänglich ist, die viele für den Medizinhistoriker interessante Nachrichten enthält s. Ullmann (1970) S. 232 f.; Sezgin (1970) S. 349f.; Brockelmann (1943) Bd. 1 S. $349 f$. und Suppl.Bd.1 (1937) S. 591.

${ }^{4}$ Frei übersetzt nach der arabischen Ausgabe des Auszugs der «Dynastiengeschichte» des Barhebraeus (Bairût 1890, S. 129, Zeile 9-15). Zur Regierungszeit des römischen Kaisers Domitius Aurelianus s. Ploetz (1960) S. 297. Die Nachricht, daß Schapur mit einem Kaiser Waffenstillstand geschlossen hat und dieser ihm seine Tochter zur Frau gab, in deren Gefolge sich auch Ärzte befanden, verzeichnet auch Ibn al-Qifț̂i (gest. 1248; arab. Edition 1903 S.133, Zeile 2-13); allerdings ist der Name des römischen Kaisers mit Philippus angegeben, wohl Philippus Arabs (reg. 244-249); Julius Lippert (Hrsg.) schreibt in der Fußnote "gemeint Valerian» (reg. 253-260) mit dem Hinweis auf einen arabischen Historiographen.

${ }^{5}$ Zur Gründung einer Akademie unter Schapur I. s. Elgood (1939) S. 1033; Ullmann (1970) S. 22; Sezgin (1970) S. 173; Kunitzsch (1975) S. 274. 
${ }^{6}$ Zitat aus Karl Hummel (1963) Sp. 2 b.

${ }^{7} \mathrm{Zu}$ Medizinschule und Krankenhaus in Gondeschapur s. Gottschalk (1965) S. 120; Art. «Gondêshâpûr » in Encyclopaedia of Islam, New Edition II (1965) Sp.1120a f.; Meyerhof (1930) S. 400-402: «Hier wurde nicht nur theoretisch - vermutlich auf Grund der Galen-Übersetzungen des Sergios - Medizin studiert, sondern ein großes Krankenhaus (Bîmâristân) diente zugleich der praktischen Ausbildung von Ärzten, und diese Einrichtung ist später für den Studiengang in der islamischen Welt vorbildlich geworden» (S. 401); Ménétrier (1931) S. 193, 198; Abdelhamid Sabra (1976) Sp. 182 b; O’Leary (1948) S. 68f.

${ }^{8} \mathrm{Zu}$ den Nestorianern s. Lexikon der Islamischen Welt (1974) Bd. 1 S. 125 f. Art. "Christen», 2. Nestorianer. Nach dem Konzil von Ephesus im Jahre 431, wo die «ZweiNaturenlehre» des Nestorius (gest. 451) verurteilt worden war, trennten sich die Anhänger des Nestorius von der Großkirche; sie wurden im byzantinischen Reich verfolgt, fanden aber im benachbarten Persien bei den Sassanidenherrschern Zuflucht. Ausführliche Darstellung bei O'Leary (1948) S. 47-62: Kap. 5 «The Nestorians», besonders 3. Abschnitt: «The Nestorian Schism» (S. 52-62); s. a. Gottschalk (1971) S. $51 \mathrm{f}$.

${ }^{9}$ Meyerhof (1930).

${ }^{10} \mathrm{Zu}$ den «Summaria Alexandrinorum» s. Ullmann (1970) S. 65-67; Sezgin (1970) S.140150; Temkin (1932) S. 66-80; Meyerhof (1930) S. 394-399; Ibn al-Qifțî (1903) S. 129, Zeile 3-16; Ibn abî Ușaibica Bd.1 (1882) S. 106, Zeile 1-108, Zeile 17. Über die Entstehungszeit der «Summaria Alexandrinorum» streiten sich die Gelehrten; sie wird vom frühen 6. bis zum späten 7. Jh. angesetzt. Nach Meyerhof (1930 S. 395): «Leider ist nun aber über die Abfassungszeit dieser Galen-Synopsis, mit der übrigens eine HippokratesSynopsis in 12 Büchern parallel läuft, gar kein Anhalt zu gewinnen.» Weiter unten heißt es bei Meyerhof (1930 S. 398): «Offenbar haben schon Hunain und sein Sohn Ishâq im IX. Jahrhundert nicht mehr viel von dem gewußt, was in Alexandrien zwei oder drei Jahrhunderte früher vorgegangen war. Daher die widersprechenden Angaben über Namen von Gelehrten, welche dem V. bis VII. Jahrhundert angehört haben. Sicher aber ergibt sich aus den kritisch gesichteten Mitteilungen der arabischen Autoren, daß kurz vor dem Eindringen des Islams in Alexandrien eine oder mehrere Schulen bestanden haben, an denen Philosophie und Medizin in bereits stark scholastischer Form gelehrt wurde.» Albert Zaki Iskandar datiert auch sehr unscharf "sixth to seventh century A.D.» (1976 S. 236, Anm. 6) und entzieht sich ebenso einer Festlegung.

${ }^{11}$ Zitat aus Temkin (1932) S. 78.

12 Zur Biographie des Sergios von Resch cAinâ s. Baumstark (1922) S. 167-169; Ullmann (1970) S. 100; Sezgin (1970) S. 177. Temkin (1932 S. 78) nennt die drei fehlenden Büchertitel auf griechisch: 1. peri hairéseôn, 2. peri sphygmôn, 3. Hygieína. Die griechischen Titel der «16 Galen-Schriften» finden sich bei Ritter von Töply (1898 S. 23f.) und bei Ritter und Walzer, die die ihnen bekannten arabischen Handschriften in Istanbuler Bibliotheken zusammenstellen (1934 S. 820-825). Albert Dietrich entdeckte in Manisa «ein schönes, leider unvollständiges Exemplar» (1966 S. 32-38), datiert auf das Jahr 1240 n. Chr. Zwar werden am Anfang die arabischen Titel aller sechzehn GalenSchriften aufgeführt, indessen nur neun behandelt; deshalb «bleiben die arabischen Texte der in den Istanbuler Hss fehlenden letzten drei Summarien ... nach wie vor unbekannt» (S. 33). 
${ }^{13}$ Zitat aus Temkin (1932) S. 79. Diesen Standpunkt vertrat offensichtlich schon zehn Jahre zuvor Anton Baumstark, der davon spricht, daß «eine Mehrzahl» der von Sergios ins Syrisch-Aramäische übersetzten Galen-Schriften «in orientalischen Ärztekreisen als Grundlage des medizinischen Studiums zu kanonischer Geltung » gelangt ist (1922 S. 168); dabei läßt Baumstark die Frage der Entstehungszeit und der Autoren offen.

${ }^{14}$ Zum Syrisch-Aramäischen als Unterrichtssprache in Gondeschapur s. Meyerhof (1926) S. 7 und (1938) S. 435; Issa (1929) S. 105; Elgood (1939) S. 1035f. und (1951) S. 48, 102; De Lacy O'Leary (1948) S. 71; Watt (1974) S. 134f.; Kunitzsch (1975) S. 273f.; Nasr (1976) S. 12, Sp. 155 a, 173; Baumstark (1922) S. 168 (vgl. Zitat in Anm. 13).

${ }^{15}$ Zur Biographie des Dschurdschîs ibn Bachtîschûc (das ist die arabisierte Namensform. des aramäischen Gewargîs bar Bochtîschô ${ }^{c}$ ) s. Ullmann (1970) S. 108 (Nr. 1); Sezgin (1970) S. 209f.; Dodge (1970) Bd. 2 S. 697, 969.

${ }^{16}$ Zur Begrüßungsepisode s. Ibn abî Ușaibica Bd. 1 (1882) S. 124, Zeile 10; Ibn al-Qifțî (1903) S. 158, Zeile 18f.; Barhebraeus (1890) S. 214, Zeile 4 f.

${ }^{17} \mathrm{Zu}$ dem «medizinischen Kompendium» des Dschurdschîs s. Ibn abî Ușaibica Bd. 1 (1882) S. 125, Zeile 31: «Sein berühmtes Kompendium; Ḥunain ibn Isḥâq hat es aus dem Syrischen ins Arabische übersetzt»; Sezgin (1970) S. 209. Bei Ibn al-Qifțî heißt es nur kurz «das Kompendium» (1903 S. 158, Zeile 6).

${ }^{18}$ Zur Biographie des Dschibra'îl s. Ullmann (1970) S. 109 (Nr. 3); Sezgin (1970) S. 226f.; Baumstark (1922) S. 227 (§ 36a).

19 Wörtliche Übersetzung aus Ibn al-Qifțî (1903) S. 134, Zeile 12-135, Zeile 10; fast wörtlich findet sich die Geschichte bei Ibn abî Ușaibica Bd. 1 (1882) S. 127, Zeile 14-31. Ob Ibn al-Qifțî mit der zitierten «Prüfung des Arztes» die heute verlorene Galen-Schrift oder die Bearbeitung dieses Themas durch Rhazes gemeint hat, wird wohl ungeklärt bleiben; vgl. bei Iskandar (1960) das Galen-Zitat bei Rhazes (S. 504, Zeile 19-505, Zeile 1) und ferner S. 509, Zeile 12-14, wo auf die intensive Beschäftigung mit dem «Analogieschlu $\beta$ » hingewiesen wird; zuerst soll der Medizinstudent über seine Fähigkeit des Disputierens und Beweisführens geprüft werden und dann hinsichtlich der Anwendung in der Heilkunde.

${ }^{20}$ Diese Schriften des Dschibra'îl werden von Ibn abî Ușaibic ${ }^{c}$ Bd. 1 (1882) S. 138, Zeile 6 f., aufgeführt.

${ }^{21}$ Zur Biographie des Yûhạnâ ibn Mâsawaih s. Ullmann (1970) S. 112-115; Sezgin (1970) S. 231-236; Art. "Ibn Mâsawayh" in Encyclopaedia of Islam, New Edition III (1971) Sp. 872 b-873a; Nasr (1976) Sp. 175 a/b.

${ }^{22}$ Wörtliche Übersetzung aus Ibn abî Ușaibica Bd. 1 (1882) S. 175, Zeile 26f. Dieser Passus findet sich fast wörtlich bei Ibn al-Qifțî (1903) S. 380, Zeile 13f. In der englischen Übersetzung des «Fihrist» durch Dodge heißt es: «He served al-Ma'mûn al$\mathrm{Mu}^{\mathrm{c}}$ tasim, al-Wâthiq, and al-Mutawakkil»(1970 S. 695); vgl. den arabischen Text im Fihrist (1348/1929-30) S. 411, Zeile 19.

${ }^{23}$ Vgl. den arabischen Text bei Ibn al-Qifțî (1903) S. 381, Zeile 19-382, Zeile 2; Ibn abî Ușaibica Bd. 1 (1882) S. 175, Zeile 31-176, Zeile 3; Barhebraeus (1890) S. 227, Zeile 13f.

${ }^{24}$ Freie Übersetzung nach Ibn Ușaibic a Bd. 1 (1882) S. 171, Zeile 31-172, Zeile 18. Yûhannâs Vater starb wahrscheinlich im ersten Viertel des 9. Jhs. - s. Sezgin (1970) S. 229.

${ }^{25}$ Wörtliche Übersetzung aus Ibn al-Qifțî (1903) S. 380, Zeile 10-13. Derselbe Passus findet sich bei Ibn abî Ușaibica Bd. 1 (1882) S. 175, Zeile 24-26. 
${ }^{26}$ Der arabische Titel «Kitâb al-muschadschdschar» bedeutet wörtlich «Buch mit Baumdarstellung». Nach Manfred Ullmann gehört es wohl zu den frühesten medizinischen Lehrbüchern, in denen das Material disjunktiv dargestellt wird (1970 S. 113); Sezgin (1970) S. 233 (Nr. 5). Ibn al-Qifțî beurteilt es als «ein wertvolles Kompendium » (1903 S. 381, Zeile 3f.; arab.: kunnâsch lahû qadr), aber bei Ibn abî Ușaibi ${ }^{c} a$ ist es nicht verzeichnet.

${ }^{27}$ Vgl. den arabischen Text bei Ibn al-Qifțî (1903) S. 382, Zeile 2.

${ }^{28}$ Zur Biographie des Ḥunain ibn Isḥâq s. Ullmann (1970) S. 115-119; Sezgin (1970) S. 247-256; Art. « Ḥunayn ibn Ishâaķ » in Encyclopaedia of Islam, New Edition III (1971) Sp. 578 b-581 a.

${ }^{29}$ Freie Übersetzung nach Ibn al-Qifțî (1903) S. 174, Zeile 6-10 und Zitat Zeile 10 (arab.: mâ li-ahli l-ḥ̂ra wa-ț-țibb).

30 Wörtliche Übersetzung aus Ibn abî Ușaibic a Bd. 1 (1882) S. 185, Zeile 6-8. Nach der rhetorischen Frage (s. Anm. 29) soll Yûhannâ in seiner Wut Ḥunain zu den Geldwechslern auf die Straße geschickt haben, also dorthin, woher er gekommen sei; dies kam einer indirekten Feuerung gleich, weshalb Ḥunain weinend die Klasse des Yûhannâ verlassen haben soll und mindestens zwei Jahre lang dort nicht mehr gesehen worden ist vgl. den arabischen Text bei Ibn abî Ușaibic a Bd. 1 S. 185, Zeile 10-14.

${ }^{31}$ Ibn abî Ușaibica spricht von 2 Jahren Abwesenheit (Bd. 1 S. 185, Zeile 14; wörtlich: fa-lam tarahû sanatain); bei Ibn al-Qifțî heißt es einmal «Jahre lang» (1903 S. 174, Zeile 14; wörtlich: sinîna), aber ein paar Zeilen weiter (S. 174, Zeile 20 f.; wörtlich: mundu talâti sinîna) «drei Jahre lang ».

32 Wörtliche Übersetzung aus Ibn al-Qifțî (1903) S. 174, Zeile 21-175, Zeile 5. Die gleiche Passage findet sich bei Ibn abî Ușaibica Bd. 1 (1882) S. 186, Zeile 1-6. Beim letzten Relativsatz lies besser mit Ibn abî Ușaibic ${ }^{\mathrm{c}}$ S. 186, Zeile 5: «Er (Sergios) ist der erste, der etwas von den Wissenschaften der Byzantiner in die syrische Sprache übersetzt hat.»

${ }^{33}$ Vgl. den arabischen Text bei Ibn al-Qifțî (1903) S. 175, Zeile 5-10; ferner Ibn abî Ușaibica Bd. 1 (1882) S. 186, Zeile 6-19.

${ }^{34}$ Zitat aus Gotthelf Bergsträsser (1925) S. 14 und vgl. den dazugehörigen arab. Text auf S. 17, Zeile 18-18, Zeile 3.

${ }^{35}$ Zitat aus Bergsträsser (1925) S. 14 und vgl. den arab. Text auf S. 18, Zeile 6-15. Widersprüchlich ist die Bemerkung, daß dieses Buch nicht in der Alexandriner Schule gelesen wurde, mit dem kurz darauffolgenden Absatz: «Dies also sind die Bücher, auf deren Lektüre man sich an der Stätte der medizinischen Lehre in Alexandria zu beschränken pflegte» (S. 15 und vgl. den arab. Text auf S. 18, Zeile 19f.).

${ }^{36}$ Zum Beispiel Bergsträsser (1925) S. 14f.: «Übersetzt hat dieses Buch (d. i. das Buch über das Heilverfahren, Nr. 20 bei Bergsträsser) aus den syrischen Exemplaren, die ich übersetzt hatte, Ḥubaiš ibn al-Ḥasan für Muḥammad ibn Mûsâ; nachdem er es übersetzt (S. 15) hatte, bat er mich dann, für ihn die letzten acht Teile zu revidieren und die Mängel, die ich darin fände, zu verbessern; diese Bitte habe ich ihm erfüllt, und zwar mit Erfolg.» Wie aus diesem Beispiel ersichtlich ist, hat Ḥunain in seinem eigenen Schriftenverzeichnis bei jeder einzelnen Schrift nach einer kurzen Inhaltsangabe Details zur Übersetzung ins Syrisch-Aramäische bzw. Arabische, über die jeweiligen Auftraggeber und eine eventuell vorhandene Übersetzung verzeichnet; s. Bergsträsser (1925).

37 Vgl. Anm. 34 . 
${ }^{38}$ Zitat aus Ullmann (1970) S. 116.

39 Wie Anm. 38.

40 Ullmann (1970) S. 118f.; Sezgin belegt insgesamt 45 selbständige Schriften Ḥunains mit einer Handschrift oder mehreren und darüber hinaus 14 weitere, deren Sachtitel uns allerdings nur aus Zitaten erhalten sind (1970 S. 249-256).

${ }^{41}$ Schipperges (1964) S. 33, 89 und (1976) S. 105f.: «Bereits im 11.Jahrhundert ist die 'Isagoge Johannitii' an den Schulen von Salerno und Chartres bekannt geworden, und sie dominiert in den scholastischen Lehrplänen bis in die Mitte des 16. Jahrhunderts.» Erst jüngst bestreitet Gregor Maurach Ḥunain die Autorschaft, indem er die Hypothese aufstellt, der Name des Verfassers, «Johannicius», ginge auf den Namen eines Byzantiners Ioanníkios zurück (1978 S. 148), so daß sich dann «die Abfolge Galen - Johannikios - Ḥunain » ergäbe (S. 149). Mir scheint als lectio facilior Johannitius der im lateinischen Mittelalter gängige Name für den nestorianischen Christen Ḥunain ibn Isḥ̂aq zu sein. Nach Manfred Ullmann hat Ḥunain «eine kleine Einleitungsschrift zur Theorie der Humoralphysiologie und -pathologie ... in drei verschiedenen Redaktionen ... verfaßt» (1970 S. 117): erstens «in disjunktiver Form », zweitens «in katechetischer Form » unter dem Titel «Masâ'il fî ț-țibb» ("Questiones medicae») und drittens «in der Form des tašğîr, d.h. in Tabellen, die den Stoff stammbaumartig ausdifferenzieren» (S.117f.). Es wird demnach in allen drei Redaktionen derselbe Stoff einer Einführung in die Mikrotekne des Galen nur in unterschiedlicher literarischer Form dargestellt.

Gregor Maurach kommt das Verdienst zu, mit seiner lateinischen, aus 12 Handschriften kollationierten Textausgabe die «abendländische Isagoge» interessierten Mediävisten offen dargelegt zu haben.

Auf einem anderen Blatt steht die Frage, ob Ḥunain der erste war, der die Medizin in zwei Teile, Theorie und Praxis, untergliederte, oder ob er möglicherweise eine byzantinische Vorlage mit dieser Zweiteilung kannte. Owsei Temkin hielt schon im Jahre 1932 in seiner «Geschichte des Hippokratismus» die Autorschaft Ḥunains für gesichert trotz mannigfaltiger verballhornter Verfassernamen in lateinischen Übersetzungen. Jüngst stellte er anhand eines Mailänder Codex, eines Kommentars des Palladius aus dem 6. Jahrhundert und eines Kommentars des Theophilus wahrscheinlich aus dem 7.Jahrhundert insofern eine gewisse Parallelität mit Ḥunain fest, als alle die Zweiteilung der Medizin in Theorie und Praxis aufweisen; bei der weiteren Untergliederung bestünde keine Übereinstimmung mehr (1977 S. 209f.). Einstweilen bleibt ungeklärt, "where in Hunain's Questions do the Alexandrians end and Hunain begin?" (s. Temkin [1977] S. 211), und ob sie oder ihre lateinische Version jemals ins Griechische übersetzt worden sind.

42 Choulant (1841) S. 399 (§ 110); Schipperges (1976) S. 106: «Das Kompendium der hochmittelalterlichen Schulmedizin ist uns als 'Ars medica' oder 'Articella' überliefert. An seinem Anfang finden wir regelmäßig die 'Isagoge in artem parvam Galeni' des Ḥunain b. Isḥâq.»

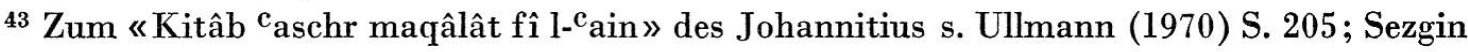
(1970) S. 251f. Max Meyerhof hat es arabisch ediert und mit einer englischen Übersetzung zugänglich gemacht (1928).

${ }^{44}$ Zur Biographie des Schaizarî (gest. 1193) s. Brockelmann (1943) Bd. 1 S. 461 (Nr. 13), Suppl. Bd. 1 S. 832 f.; Schaizarîs Manuale trägt den Sachtitel «Nihâyat ar-rutba fî țalab 
al-hiisba » (wörtl.: Schlußstufe bei der Bewerbung um die Marktaufsicht); es ist auszugsweise von Nicola Ziyadeh arabisch ediert worden (1962); vgl. daselbst den Passus über die Augenärzte (S. 101, Zeile 14ff.).

${ }^{45}$ Max Meyerhof hat anhand der Angaben des Ibn abî Ușaibica den jeweiligen Anteil nestorianisch-christlicher, sabäischer, jüdischer und muslimischer Ärzte vom 9. bis 11. Jahrhundert festgestellt (1929 S. 116f.):

\begin{tabular}{rrllc}
\hline Ärzte & nestorianische & Sabier & jüdische & muslimische \\
\hline 9. Jh. & 130 & 3 & 3 & 5 \\
10. Jh. & 29 & 4 & 6 & 30 \\
11. Jh. & 4 & - & 7 & Vielzahl \\
\hline
\end{tabular}

${ }^{46}$ Zur Biographie des Dschâhiz (776/7-869), eines der besten Stilisten der arabischen Literatur, s. Lexikon der islamischen Welt (1974) Bd. 1 S. 147.

${ }^{47}$ Freie Übertragung aus dem «Buch über die Geizhälse» (Kitâb al-buchalâ'), hrsg. v. van Vloten (1900) S. 109, Zeile 17-110, Zeile 5, bzw. hrsg. v. Ḥâdschirî (1976) S. 102, Zeile 10-16.

${ }^{48}$ Zum Krankenhaus des Hârûn ar-Raschîd s. Issa (1929) S. 94f., 175; Art. «Bîmâristân » in Encyclopaedia of Islam, New Edition I (1960) Sp. 1223 a; Gottschalk (1965) S. 120 und (1971) S. 125; Hamarneh (1962) S. 368; Watt (1972) S. 36 und (1974) S. 227.

${ }^{49}$ Zum «Bait al-hikma» s. Encyclopaedia of Islam, New Edition I (1960) Sp. 1141 a; Schipperges (1976) S. 62, 63-65; Gottschalk (1965) S. 122f.; Sabra (1976) S. 181.

${ }^{50}$ Zur Biographie des Sâbûr ibn Sahl s. Fihrist (1348/1929-30) S. 413, Zeile 16-21; Dodge (1970) S. 698, 1085f.; Ibn abî Ușaibica Bd. 1 (1882) S. 161, Zeile 15-22; Ibn al-Qifț̂i (1903) S. 207, Zeile 3-8; Barhebraeus (1890) S. 255, Zeile 13-17; Issa verzeichnet ihn als Direktor des Krankenhauses in Gondeschapur (1929 S. 108, nr. 8). Ibn abî Ușaibicia gibt den Umfang des "Großen Aqrabadin» mit 17 Kapiteln an (Bd. 1 S. 171, Zeile 19), die anderen arabischen Quellen dagegen mit 22 Kapiteln; möglicherweise gab es eine umfangreichere (22 Kap.) und eine kürzere redaktionelle Bearbeitung (17 Kap.). Vgl. Elgood (1951) S. 50, 92 f., 175.

\section{Literaturverzeichnis}

Barhebraeus (Ibn al-' Ibrî): Ta'rîch muchtașar ad-duwal. Hrsg. v. Anțûn Șâlhânî. Bairût 1890.

Baumstark, Anton, Geschichte der syrischen Literatur mit Ausschließung der christlichpalästinensischen Texte. Bonn 1922. Photomech. Nachdr. Berlin: de Gruyter 1968.

Bergsträsser, Gotthelf: Ḥunain ibn Ishâq. Über die syrischen und arabischen GalenÜbersetzungen. Zum ersten Mal hrsg. u. übers. Leipzig 1925 (= Abhandlungen der Kunde des Morgenlandes, Bd. XVII, 2). Kraus-Reprint, Nendeln 1966.

Bosworth, Clifford Edmund, The Islamic Dynasties - Chronological and Genealogical Handbook. Edinburgh: University Press 1967 (= Islamic Surveys 5).

Brockelmann, Carl, Geschichte der arabischen Litteratur. Bd. 1.2. 2. Aufl. Leiden: Brill 1943, 1949. Suppl.1-3. Leiden 1937-1942. 
Choulant, Ludwig, Handbuch der Bücherkunde für die ältere Medicin. 2. umgearb. u. verm. Aufl. Leipzig 1841.

Dictionary of Scientific Biography. Ed. by Charles Coulston Gillispie. Vol. $1 \mathrm{ff}$. New York: Ch. Scribner's Sons $1970 \mathrm{ff}$.

Dietrich, Albert, Medicinalia Arabica. Studien über arabische medizinische Handschriften in türkischen und syrischen Bibliotheken. Göttingen: Vandenhoek \& Ruprecht 1966 (= Abhandlungen der Akademie der Wissenschaften in Göttingen. Phil.-hist. Kl. Dritte Folge, 66).

Dodge, Bayard (Ed. and Tr.), The Fihrist of al-Nadîm. A tenth-century survey of muslim culture. Vol. 1.2. New York, London: Columbia University Press 1970 (= Records of Civilization. Sources and studies. No. LXXXIII).

Elgood, Cyril, A Medical History of Persia and the Eastern Caliphate from the earliest times until the Year A.D.1932. Cambridge: University Press 1951.

ders., Jundi Shapur - A Sassanian university. In: Proceedings of the Royal Society of Medicine 32 (1938/39), S. 1033-1036.

Encyclopaedia of Islam. New Edition. Vol. I ff. London, Köln: Luzac, Brill $1960 \mathrm{ff}$.

Fihrist s. Dodge, Bayard.

ders. s. Ibn an-Nadîm.

Gottschalk, Hans L., Die Kultur der Araber. In: Handbuch der Kulturgeschichte 2. Abt. Frankfurt: Athenaion 1971, S. 9-205.

ders., Die Rezeption der antiken Wissenschaften durch den Islam. In: Anzeiger d. österr. Akad.d. Wissenschaften. Phil.-hist. Kl. 102 (1965), Nr. 7, S. 111-135.

Hamarneh, Sami Khalaf, Development of Hospitals in Islam. In: Journal of the History of Medicine 17 (1962), No. 3, S. 366-384.

Hummel, Karl, Die Anfänge der iranischen Hochschule Gundischapur in der Spätantike. In: Tübinger Forschungen Nr. 9 (1963), S. 1-4.

Ḥunain ibn Ishâq s. Bergsträsser, Gotthelf.

ders. s. Meyerhof, Max, The Book of the ten Treatises.

Ibn abî Ușaibica: 'Uyûn al-anbâ' fî țabaqât al-ațibbâ'. Vol. I/II. Hrsg. v. August Müller. Kairo, Königsberg 1882-1884.

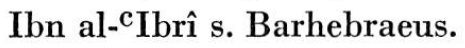

Ibn an-Nadîm: al-Fihrist. Mișr 1348 Hidschra (= Kairo 1929/30).

Ibn al-Qifțî: Ta'rîch al-ḥukamâ' wa-huwa mutchtașar az-Zauzanî. Hrsg. v. Julius Lippert. Leipzig 1903.

Iskandar, Albert Zaki, An attempted reconstruction of the late Alexandrian medical curriculum. In: Medical History 20 (1976), S. 235-258.

ders., ar-Râzî wa-miḥnat aț-țabîb. In: al-Mašriq 54 (1960), S. 471-522 (arab.).

Issa, Ahmed, Histoire des Bimaristans (Hôpitaux) à l'époque Islamique. In: Comptes Rendus du Congrès International de Médecine et Hygiène Tropique, Caire 1929, S.81209.

Kunitzsch, Paul, Über das Frühstadium der Aneignung antiken Gutes. In: Saeculum 26 (1975), S. 268-282.

Lexikon der Islamischen Welt. Hrsg. v. Klaus Kreiser, Werner Diem, Hans Georg Majer. Bd. 1.2.3. Stuttgart u. a.: Kohlhammer 1974 (= Urban TB 200/1-3).

Lippert, Julius (Hrsg.) s. Ibn al-Qifțî. 
Maurach, Gregor, Johannicius: Isagoge ad Techne Galieni. In: Sudhoffs Archiv 62 (1978), Nr. 2, S. 148-174.

Ménétrier, M.P., La médecine Arabe, son Rôle dans l'histoire et son influence sur la médecine française. In: Bulletin de la Société française d'histoire de la médecine 25 (1931), S. 191-207.

Meyerhof, Max, Von Alexandrien nach Bagdad. Ein Beitrag zur Geschichte des philosophischen und medizinischen Unterrichts bei den Arabern. In: Sitzungsberichte der Preuß. Akad. d. Wiss. 1930. Phil.-hist. Kl. Berlin 1930, S. 389-429.

ders.: The Book of the ten Treatises on the Eye Ascribed to Ḥunain ibn Ishâkş. Engl. transl. Cairo 1928.

ders., Notes sur quelques médecins juifs égyptiens qui se sont illustrés à l'époque arabe. In: Isis 12 (1929), S. 113-131.

ders., Mediaeval Jewish Physicians in the Near East, from Arabic sources. In: Isis 28 (1938), S. 432-460.

ders., Les Versions Syriaques et Arabes des Ecrits Galéniques. In: Byzantion 3 (1926), S. 3-21.

Müller, August (Hrsg.) s. Ibn abî Ușaibica.

Nasr, Seyyid Hossein, Islamic Science. An Illustrated Study. Westerham, Kent: World of Islam Festival Publishing Company Ltd. 1976.

O'Leary, De Lacy, How Greek science passed to the Arabs. London: Kegan Paul 1948.

Ploetz, Karl, Auszug aus der Geschichte. 26. Aufl. Würzburg: Ploetz-Verl. 1960.

Ritter, Helmut und Richard Walzer, Arabische Übersetzungen griechischer Arzte in Stambuler Bibliotheken. In: Sitzungsberichte der Preuß. Akad. d. Wiss. Phil.-hist. Kl. 1934, Bd. XXVI, S. 1-48.

Sabra, Abdelhamid I., Philosophie und Naturwissenschaften. Der islamische Beitrag zur Entwicklung der Wissenschaft. In: Welt des Islam. Hrsg. v. Bernard Lewis (Deutsche Übers.). Braunschweig: Westermann 1976, S. 181-200.

Schaizarî, ${ }^{c}$ Abd ar-Rahmmân s. Ziyadeh, Nicola.

Schipperges, Heinrich, Die Assimilation der arabischen Medizin durch das lateinische Mittelalter. Wiesbaden: Steiner 1964 (= Sudhoffs Archiv. Beihefte 1964, Heft 3).

ders. Eine griechisch-arabische Einführung in die Medizin. In: Deutsche Medizinische Wochenschrift 87 (1962), Nr. 34, S. 1675-1680.

ders., Arabische Medizin im lateinischen Mittelalter. Berlin, Heidelberg, New York: Springer 1976 (= Sitzungsberichte der Heidelberger Akad. d. Wiss. Math.-naturwiss. Kl. 1976, 2. Abh.).

Senn, Gustav, Die Entwicklung der biologischen Forschungsmethode in der Antike und ihre grundsätzliche Förderung durch Theophrast von Eresos. Aarau 1933 (= Veröffentlichungen der Schweizerischen Gesellschaft für Geschichte der Medizin und der Naturwissenschaften, VIII).

Sezgin, Fuat, Geschichte des arabischen Schrifttums. Bd. III. Medizin - Pharmazie - Zoologie - Tierheilkunde bis ca. 430 H. (= 1038/9 n. Chr.). Leiden: Brill 1970.

Temkin, Owsei, The Double Face of Janus and Other Essays in the History of Medicine. Baltimore, London: The Johns Hopkins University Press 1977.

ders., Geschichte des Hippokratismus im ausgehenden Altertum. In: Kyklos 4 (1932), S. 1-80. 
Töply, Robert Ritter von, Studien zur Geschichte der Anatomie im Mittelalter. Leipzig, Wien: Deuticke 1898.

Ullmann, Manfred, Die Medizin im Islam. Leiden: Brill 1970 (= Handbuch der Orientalistik. 1. Abt. Erg.-bd. VI, 1.Abschn.).

Vloten, G. van, Le livre des avares par Abou Othman Amr Ibn Bahr al-Djahiz de Basra. Leyde: Brill 1900 (arab. Ed.).

Watt, Montgomery, The Influence of Islam on Medieval Europe. Edinburgh: University Press 1972 (= Islamic Surveys 9).

ders., The Majesty that was Islam. The Islamic World 661-1100. London: Sidgwick \& Jackson 1974.

Ziyadeh, Nicola: Al-Ḥisba wal-Muḥtasib fil-Islâm. Bairût 1962 (= Nuṣ̂ṣ wa-durûs 12).

\section{Summary}

The medical School of Jundishapur in Persia flourished from the 6 th to the 9 th century. The Arabs conquered the town in 736. Greek medicine came from Alexandria to Baghdad through Jundishapur which was the starting-point of Arabic medicine. Great physicians of the School were Jurjîs (died 768) whose grandson became the physician of the caliph Harun al-Rashid, then Yûhannâ called Mesuë the elder (777-857) and his pupil, the Nestorian teacher Hunain or Johannitius, the indefatigable translator of Hippocrates, Galen, and other Greek authors. The last work of the School was a pharmacopoeia written in 869 .

Dr.phil. Friedrun Roswitha Hau

Medizinhistorisches Institut

Sigmund-Freud-Straße

D-5300 Bonn 1 